\title{
PEIXE-BOI-DA-AMAZÔNIA: INFORMAÇÕES PARA O DESMAME EM CATIVEIRO
}

\section{AMAZON MANATEE: INFORMATION WEANING IN CAPTIVITY}

\author{
Bruno Barbosa Iespa ${ }^{1}$, José Milton Barbosa ${ }^{2}$, Kátia Cristina de Araújo Silva $^{3}$ \& Israel Hidenburgo Aniceto Cintra ${ }^{4}$ \\ ${ }^{1}$ Centro Nacional de Pesquisa e Conservação da Biodiversidade Marinha do Norte, Instituto Chico Mendes de \\ Conservação da Biodiversidade - ICMBio \\ ${ }^{2}$ Departamento de Engenharia de Pesca e Aquicultura, Universidade Federal de Sergipe - UFS \\ ${ }^{3-4}$ Instituto Socioambiental e dos Recursos Hídricos, Universidade Federal Rural da Amazônia - UFRA \\ *E-mail: israel.cintra@ufra.edu.br
}

\section{RESUMO}

Nesta revisão coletou-se informações sobre o peixeboi-da-amazônia acerca do desmame em cativeiro e posterior introdução no ambiente natural: sistemática, distribuição, $\quad$ reprodução, alimentação, comportamento em cativeiro, importância ecológica, econômica e cultural e legislação para o manejo em cativeiro.

Palavras-chave: Trichechus inunguis, alimentação, importância ecológica, legislação.

\begin{abstract}
In this review we collected information on the Amazonian manatee about weaning in captivity and subsequent introduction into the natural environment: systematic, distribution, reproduction, feeding, behavior in captivity, ecological, economic and cultural importance and legislation for management in captivity.
\end{abstract}

Key words: Trichechus inunguis, food, ecological importance, amazonian manatee. 


\section{Introdução}

A ordem Sirênia Illiger, 1811, é constituída por duas famílias, Trichechidae Gill, 1872 e Dugongidae Gray, 1821. A família Dugongidae é constituída pelas espécies Dugong dugon (Müller, 1776) e Hydrodamalis gigas (Zimmermann, 1780), sendo que H. gigas foi extinta no final do século XVIII. A família Trichechidae abriga o gênero Trichechus Linnaeus, 1758 e as espécies Trichechus inunguis (Natterer, 1883), Trichechus manatus Linnaeus, 1758 e Trichechus senegalensis Link, 1795. Assim, existem na natureza quatro espécies viventes: o peixe-boi-da-amazônia $T$. inunguis, o peixe-boi-marinho $T$. manatus, o peixe-boi-africano T. senegalensis e o dugongo D. dugon (Itis, 2020). No Brasil ocorrem duas espécies de Sirênios: o peixe-boi-marinho e o peixe-boi-da-amazônia (Luna et al., 2011).

O peixe-boi-da-amazônia é o menor dos peixes-boi, sendo essencialmente fluvial. Tem como característica a ausência de unhas nas nadadeiras peitorais, o que gerou o nome específico inunguis (do latim, sem unhas) e apresenta uma mancha branca irregular na região ventral que pode estar ausente em alguns animais (Rosas, 1994).

O peixe-boi-da-amazônia é o mamífero aquático mais caçado do Brasil, e, embora em menor intensidade do que no início do século XX (Luna et al., 2011), seu status de conservação está classificado como "vulnerável" na lista das Espécies Ameaçadas da Fauna e Flora da União Internacional para Conservação da Natureza (IUCN, 2012). De forma que a sua reprodução em cativeiro, para soltura no ambiente natural, tem sido uma estratégia exitosa no Brasil.

Apesar disso, as informações necessárias para subsidiar a soltura de Sirênios que vivem em cativeiros são incipientes. Como exemplo, é possível observar que há carência de estudos sobre o comportamento que visam identificar estereotipias de cativeiro que são considerados importantes parâmetros para promover a saúde e conservação das espécies (Anzolin, Carvalho, Viana-Júnior, Normande \& Souto, 2013), pois ao permitir a identificação de aspectos mais difíceis de serem percebidos na natureza, nos possibilita maior conhecimento da sua biologia, além de ser um procedimento obrigatório, como determina a Instrução Normativa $\mathrm{n}^{\mathrm{0}}$ 3/2002 do Ibama que trata de instalações e manutenção de mamíferos aquáticos em cativeiro (Brasil, 2002).

No Brasil, existem instituições com expertise para manter e reabilitar filhotes de peixes-boi órfãos em cativeiro: Laboratório de Mamíferos Aquáticos (LMA) do Inpa em Manaus; o Centro de Pesquisas e Preservação dos Mamíferos Aquáticos (CPPMA), ligado a Manaus Energia S.A., em Balbina (AM); Zoológico de Santarém (Zoofit-PA); Centro de Mamíferos Aquáticos (CMA), em Itamaracá (PE) e o Centro de Mamíferos Aquáticos/ICMBio, em Belém, que vinha resgatando e reabilitando em cativeiro filhotes órfãos de peixes-boi-da-amazônia, já tendo este centro de pesquisa, inclusive, realizado a soltura de dois animais (um macho e uma fêmea) em uma Unidade de Conservação Federal (Reserva Extrativista TapajósArapiuns), próximo à cidade de Santarém-PA, em setembro de 2007 (Luna et al., 2011).

Nesta revisão coletou-se informações sobre o peixe-boi-da-amazônia Trichechus inunguis (Natterer, 1883) acerca do desmame em cativeiro e posterior introdução no ambiente natural.

\section{Sistemática}

A classificação dos mamíferos pode ser discutida em duas bases, sendo os caracteres morfológicos e moleculares divisores das linhas de atuação de pesquisadores. A linha de pesquisa, com base nos caracteres moleculares, aponta para o fato de cetáceos serem mais aparentados dos morcegos que dos peixes-boi-daamazônia, contrapondo com a teoria morfológica. A ideia de incluir os peixes-boi-da-amazônia na divisão Ungulata, onde os Cetáceos formam com os Sirênios um grupo monofilético de ancestral comum confronta com a separação observada em cladograma de teoria molecular. Porém, mesmo considerando as duas linhas de sistemas de classificação, observa-se que os peixes-boi compõem uma ordem que teve um ancestral comum aos elefantes, sendo os hyrax amarelo-manchado Heterohyrax brucei (Gray, 1868) um "parente mais próximo" do peixe-boi-da-amazônia que um boto tucuxi Sotalia fluviatilis (Gervais e Deville, 1853), cetáceo que convive no mesmo hábitat que T. inunguis (Pough, 2003).

Segundo Itis (2020) a hierarquia taxonômica do peixe-boi-da-amazônia é a seguinte: Reino Animalia, Sub-reino Bilateria, Infra-reino Deuterostomia, Filo Chordata, Subfilo Vertebrata, Infra-filo Gnathostomata, Superclasse Tetrapoda, Classe Mammalia Linnaeus, 1758, Subclasse Theria Parker and Haswell, 1897, Infra-classe Eutheria Gill, 1872, Ordem Sirenia Illiger, 1811, Família Trichechidae Gill, 1872, Gênero Trichechus Linnaeus, 1758, Espécie Trichechus inunguis (Natterer), 1883. 


\section{Distribuição}

Genericamente, o peixe-boi-da-amazônia $T$. inunguis é encontrado na parte sul do continente americano (Itis, 2020). A espécie é endêmica da bacia do rio amazonas, onde se distribui pelos principais afluentes e lagos, desde o Peru, Colômbia e Equador até a foz, no Atlântico, ao redor da Ilha do Marajó. No Brasil, ocorre em todas as bacias dos principais rios da Amazônia sendo sua distribuição limitada por cachoeiras e corredeiras (Luna et al., 2011).

\section{Reprodução}

A reprodução destes animais está relacionada com o ciclo de cheia e vazante da bacia amazônica, com o acasalamento e os nascimentos ocorrendo durante a cheia (dezembro-junho), tendo como pico de nascimentos no período de fevereiro a maio. Nesse período, há maior disponibilidade de alimentos (macrófitas), o que favorece a reposição energética demandada durante os primeiros meses de lactação (Cintra, 2004). A idade em que o peixe-boi da Amazônia está apto à participar do processo reprodutivo, assim como o intervalo entre nascimentos, não estão bem definidos, embora as estimativas apontem que a maturidade sexual ocorra a partir do quinto ano, com intervalo de três anos entre nascimentos (Silva, 2004), originando apenas um filhote por gestação (Best, Ribeiro, Yamakoshi \& Silva, 1982; Best, 1984).

\section{Alimentação}

No contexto da reintrodução dos peixes-boi é importante realizar alterações na alimentação dos animais, visando prepará-los para a vida no ambiente natural (Anzolin, Carvalho, Viana-Júnior, Normande \& Souto, 2013), sendo o advento do desmame uma das etapas desse processo.

O peixe-boi-da-amazônia é um animal exclusivamente herbívoro, que na natureza se alimenta especialmente de plantas emergentes, representadas principalmente pela Família das Gramíneas (Colares \& Colares, 2002). Em cativeiro, os filhotes são alimentados com fórmulas artificiais fornecidas com utilização de mamadeiras (Figura 1), com plantas dos gêneros Brachiaria, Halodule, Gracilaria, Cryptonema, dentre outras, além de fruto ou frutas, legumes e verduras (Colares, Colares, Bianchini \& Santos, 2000; Pantoja, Rosas, Silva \& Santos, 2010; Anzolin, Carvalho, Viana-Júnior, Normande \& Souto, 2013; Barbosa, Silva \& Pereira Júnior, 2013).

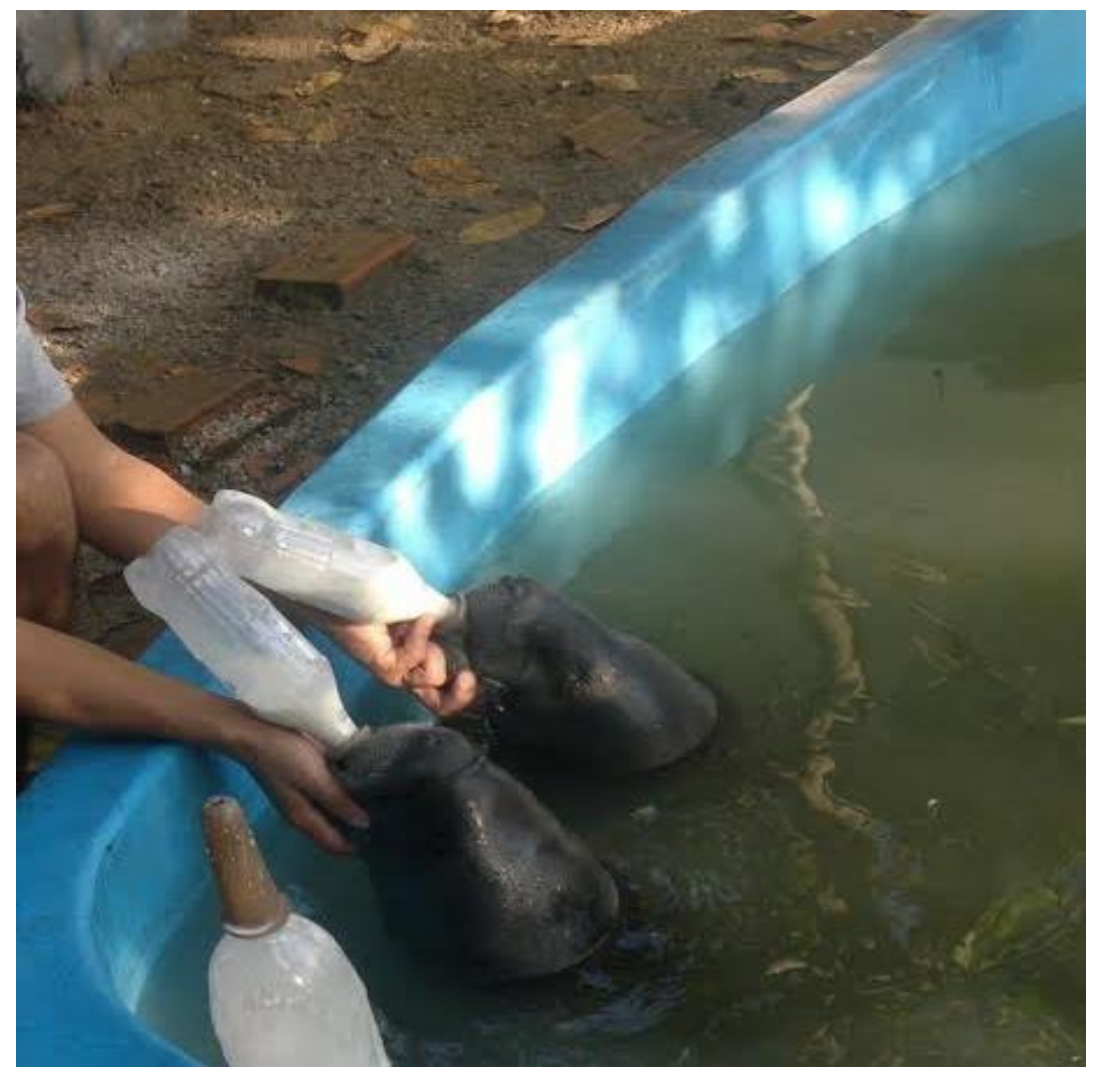

Figura 1. Exemplares Trichechus inunguis, alimentados com mamadeiras na base do Centro de Mamíferos Aquáticos/ICMBio. 
Colares e Colares (2002) ao avaliarem amostras estomacais e fezes de T. inunguis em rios e lagos da Amazônia Central, identificaram Paspalum repens (P. J. Bergius), Echinochloa polystachya (Kunth) e Eichornia crassipes (Mart.) Solms, como as três espécies mais frequentes em sua alimentação, sugerindo que no período de águas baixas, quando o alimento é mais escasso, os animais apresentaram maior diversidade de espécies em sua dieta, enquanto que na cheia, apresentaram dieta mais seletiva. E. crassipes é um importante componente da alimentação dos T. manatus da Flórida (EUA) e tende a se proliferar muito em barragens, da mesma forma que Pistia stratiotes L., que é considerada invasora em muitos países e conhecida como forrageira para peixe-boi (Guterres, Marmontel, Ayub, Singer \& Singer, 2008).

\section{Comportamento em cativeiro}

Antes da reintrodução de peixes boi em ambientes naturais é indispensável que haja um monitoramento pré-soltura dos animais, visando inferir a eficácia do trabalho, no sentido de avaliar a capacidade de ajuste do indivíduo ao novo ambiente e as chances de interação com outros animais (Medina, 2008). A mesma autora relata que o estudo de animais em cativeiro é importante também na determinação de padrões comportamentais, tanto individuais como associativos, possibilitando a constatação de associações entre indivíduos que poderiam persistir após liberação na natureza, sendo importante detectar comportamentos individuais e estereotipias que dificultem o sucesso do processo de reintrodução.

É notório que o estudo do comportamento animal é pautado pela contribuição que os etologistas têm ao descrever características gerais de estímulo-resposta. Os estudos tendem a enriquecer o catálogo de comportamentos observados em grande variedade de animais, sob distintas condições, além de reforçar a importância de forças interativas, ecológicas e evolutivas que causam os distintos comportamentos (Nascimento, Medeiros \& Yamamoto, 2008).

O estudo do comportamento animal não é um importante campo científico apenas por si próprio, mas também por ter feito importantes contribuições para outras disciplinas e se mostram relevantes para conservação de espécies ameaçadas de extinção visando a reintrodução de animais e para promover o bemestar animal (Snowdon, 1999).

Descrevendo o comportamento de superfície do boto-cinza, Sotalia guianenses (P.-J. van Bénéden, 1864), na praia da Pipa, no estado do Rio Grande do Norte, Nascimento, Medeiros e Yamamoto (2008) relatam que no estudo de comportamento animal, o etograma, que consiste na descrição de forma detalhada de comportamentos realizados por determinada espécie, é a base para estudos de comportamento animal de espécies pouco conhecidas, como também permite comparar comportamentos de populações distintas.

No universo dos Sirênios, Anzolin, Carvalho, Viana-Júnior, Normande e Souto, (2013) ressaltam haver poucos estudos publicados sobre os diferentes comportamentos de T. manatus em cativeiro, fazendo referência e comparando quatro comportamentos, classificados como estereotipias, realizados por espécimes cativas de três localidades.

A busca por trabalhos sobre o comportamento de T. inunguis remete as pesquisas com animais do mesmo gênero, como por exemplo o etograma elaborado por Medina (2008) quando estudou T. manatus cativos. O repertório de comportamentos amostrados no estudo de Medina (2008) serviu de base para o trabalho de Barreto (2011), quando analisou os comportamentos de exemplares de $T$. inunguis cativos em Belém, estado do Pará.

O comportamento durante o desmame de sete exemplares de $T$. inunguis em cativeiro foi reportado por Iespa, Ribeiro, Barreto, Klautau e Cintra (2017), quando os autores compararam os padrões de alguns comportamentos antes (mamando), durante (desmamando) e depois (herbivoria) da fase de desmame.

\section{Importância ecológica, econômica e cultural}

O peixe-boi-da-amazônia possui importância ecológica no equilíbrio do ecossistema, pois suas fezes fertilizam as águas, auxiliando na produção de alimentos para outras espécies (Silva \& Marmontel, 2009). Por outro lado, contribuem significativamente para o controle de plantas aquáticas macrófitas, na ecologia de leitos de algas e na produção de algas e plânctons (Domning \& Magor, 1977). Ademais, estes animais também desenvolveram importante papel no desenvolvimento da região amazônica, sendo inicialmente abatido por tribos indígenas para utilização de sua carne e como matéria-prima de produtos de valor agregados, tais como, manteiga, óleo e couro, além da carne fresca, seca, frita e conservada em óleo (michira) para comunidades ribeirinhas (Best, 1984). Assim, foi alvo de caça em grande escala durante o século passado. Nas regiões denominadas de "Pesqueiro Real de Peixes Boi", onde atualmente é o município de Faro (oeste do Pará), a caça indiscriminada, com utilização de arpões, sem distinção de 
tamanho das presas, associada ao longo tempo de reprodução e de gestação e o fato de gerarem um ou dois filhotes por ano, resultou na ausência de peixes-boi-da-amazônia em lagos e outros ambientes, onde antes eram abundantes (Ferreira, 1903).

T. inunguis é alvo de uma das principais manifestações culturais da cidade de Novo Airão, no Amazonas, à cerca de $200 \mathrm{~km}$ de Manaus. Semelhante ao festival dos bois de Parintins-AM, com a disputa entre Caprichoso e Garantido, o "Festival do Peixe Boi", tem seu ponto culminante na disputa por meio de desfile de fantasias, coreografias e enredos, entre dois grupos: Jaú e Anavilhanas. Esse festival teve início em 1989, concomitantemente com o protesto contra a proibição da caça do peixe-boi e demais restrições advindas da criação das unidades de conservação federais: Parque Nacional do Jaú-AM, criado em 1980 e da Estação Ecológica de Anavilhanas-AM criada em 1981.

\section{Legislação para o manejo em cativeiro}

A legislação brasileira que regula a manutenção de Sirênios em cativeiro, existem regras previstas na Instrução Normativa do IBAMA no 3 de 8 de fevereiro de 2002, que regulam as instalações destinadas à manutenção e alimentação destes animais que devem ser adotadas por empresas e instituições públicas ou privadas. Dentre os principais aspectos a serem considerados estão: a volumetria dos recintos para $T$. inunguis, que pode variar de 100 a $700 \mathrm{~m}^{3}$ por animal, sendo acrescentado $30 \mathrm{~m}^{3}$ para cada animal extra. Tal variação se deve à classificação do tipo de atividade que estiver sendo desenvolvida pela entidade mantenedora dos animais. A referida normatização classifica os tipos de recintos de acordo com o tipo de manejo que será realizado: recinto/piscina para indivíduos manejados de forma permanente $\left(700 \mathrm{~m}^{3}\right.$ para até dois indivíduos); recintos/piscinas para reabilitação, que é o local em que o animal permanece sob cuidados veterinários intensivos, visando sua soltura ou destinação adequada $\left(100 \mathrm{~m}^{3}\right.$ para um indivíduo, sendo acrescidos $30 \mathrm{~m}^{3}$ para cada indivíduo extra); recintos/piscinas de quarentena, local destinado a abrigar animais recém chegados em observação, antes de sua integração ao plantel (Brasil, 2002).

A legislação em questão também enfatiza a necessidade de que os programas de reabilitação realizem a observação permanente do comportamento dos animais, assim como a realização frequente de exames de qualidade de água, estabelecendo a manutenção do $\mathrm{pH}$ da água necessariamente entre 7,2 e 8,4. Para cativeiros de T. inunguis, assim como para os demais cativeiros de mamíferos aquáticos de água doce, as variáveis biológicas deverão ser adequadas à balneabilidade.

\section{Recomendações}

A utilização das três espécies vegetais Pistia stratiotes L., Eichornia crassipes (Mart.) Solms e Hymenachne amplexicaulis (Rudge) Nees, visando efetivar o desmame e manter em herbivoria plena os exemplares de $T$. inunguis em cativeiro se mostra nutricionalmente inadequado.

O papel do tratador de animais é indispensável na percepção dos problemas que porventura surjam com os animais. O tratador de animais é comparável ao termômetro que indica quando uma criança está febril. Ele é o primeiro a detectar quando o animal está com problemas.

\section{Referências}

Anzolin, D.G., Carvalho, P.S.M., Viana-Júnior, P.C., Normande, I.C. \& Souto, A.S. (2014) Stereotypical behaviour in captive West Indian manatee (Trichechus manatus). Journal of the Marine Biological Association of the United Kingdom, 94(6), 1-5.

Barbosa, P.S., Silva, V.M.F. \& Pereira-Júnior, G. (2013) Tempo de passagem de duas dietas no trato gastrointestinal do peixe-boi da Amazônia Trichechus inunguis (Natterer, 1883) em cativeiro, Acta Amazônica, 43(3), 365-370.

Barreto, U.H.A. (2011) Análise comportamental de peixe-boi amazônico (Trichechus inunguis) criado em cativeiro no centro de mamíferos aquáticos. Trabalho de Conclusão de Curso (Bacharelado em Medicina Veterinária), Universidade Federal Rural da Amazônia, Belém, PA, Brasil.

Best, R.C. (1984) The aquatic mammals and reptiles on the Amazon. In: H. Sioli (Ed.). The Amazon limnology and landscape ecology of a mighty tropical river and its basin (pp. 371-412).

Best, R.C., Ribeiro, G.A., Yamakoshi, M. \& Silva, V.M.F. (1982) Artificial feeding for un weaned Amazonian manatees. International Zoo Yearbook, 22(1), 263-267. 
Brasil. Ministério do Meio Ambiente. Ibama. Instrução normativa do Ibama $\mathrm{n}^{\mathrm{o}} 3$, de 8 de fevereiro de 2002. Estabelece critérios para manutenção em cativeiro das espécies de mamíferos aquáticos. Diário Oficial da União, Brasília, DF, 13 fev. n⿳ํㅡㄹ. Seção 1, pp. 65-67, 2002.

Cintra, R. (2004) História natural ecologia e conservação de algumas espécies de plantas e animais da Amazônia. Manaus: Edua/Inpa/Fapeam.

Colares, I.G. \& Colares, E.P. (2002) Food plants eaten by Amazonian Manatees (Trichechus inunguis, Mammalia: Sirenia), Brasilian Archives of Biology and Tecnology, 45(1), 67-72.

Domning, D.P. \& Magor, D.M. (1977) Taxa de substituição horizontal de dentes no peixe-boi, Acta Amazônica, 7(3), 435-438.

Ferreira, A.R. Memória sobre o peixe-boi e o uso que lhe dão no estado do Grão Pará, Arquivo Museu Nacional Rio de Janeiro, 12. p. 169-174, 1903. Disponível em em 26 de novembro de 2012. http://www.obrasraras.museunacional.ufrj.br/o/0017/169-174.pdf>.

Guterres, M.G., Marmontel, M., Ayub, D.M., Singer, R.F. \& Singer, R.B. (2008) Anatomia e morfologia de plantas aquáticas da Amazônia (utilizadas como potencial alimento por peixe-boi amazônico). $1^{\mathrm{a}}$ ed. p. 44-150.

Iespa, B.B., Ribeiro, A.S.S., Barreto, U.H.A., Klautau, A.G.C. M. \& Cintra, I.H.A. (2017) Comportamento durante o desmame de peixe-boi-da-amazônia Trichechus inunguis (Natterer, 1883) em cativeiro. Revista Brasileira de Saúde e Produção Animal, 18(1), 183-192.

ITIS. Integrated Taxonomic Information System. Disponível em 20 de agosto de 2020. http://www.itis.gov

IUCN. União Internacional para Conservação da Natureza. Lista Vermelha de Espécies Ameaçadas. Versão 2.012,2. Disponível em 27 de novembro de 2012. http://: < www.iucnredlist.org >. Acesso em.

Luna, F.O., Silva, V.M.F., Andrade, M.C.M., Marques, C.C., Normande, I.C., Veloso, T.M.G. \& Severo, M.M. (2011) Plano de ação nacional para a conservação dos Sirênios: peixe-boi-da-Amazônia: Trichechus inunguis e peixe-boi-marinho: Trichechus manatus, Série Espécies Ameaçadas, Brasília: Instituto Chico Mendes de Conservação da Biodiversidade, ICMBio.

Medina, V.E.H. (2008) Comportamento do peixe-boi (Trichechus manatus manatus) nos oceanários de Itamaracá: manejo e condições abióticas. Dissertação de Mestrado, Universidade Federal de Pernambuco, Recife, PE, Brasil.

Nascimento, L.F., Medeiros, P.I.A.P. \& Yamamoto, M.E. (2008) Descrição do comportamento de superfície do boto cinza, Sotalia guianensis, na Praia da Pipa - RN. Psicologia: Reflexão e Crítica, 21(3), 509-517.

Pough, F.H., Janis, C.M. \& Meser, J.B. (2003) A vida dos vertebrados. $3^{\circ}$ edição. Capítulo XIX. pp. 539542 .

Pantoja, T.M.A., Rosas, F.C.W., Silva, V.M.F. \& Santos, A.M.F. (2010) Urinary parameters of Trichechus inunguis (Mammalia, Sirenia): reference values for the Amazonian Manatee, Brazilian Journal of Biology, 70(3), 607-615.

Rosas, F.C.W. (1994) Biology, conservation and status of the Amazonian Manatee Trichechus inunguis, Mammal Review, 24(2), 49-59.

Silva, V.M.F. (2004) O peixe-boi da Amazônia Trichechus inunguis (Sirenia: Trichechidae), In: R. Cintra (Ed.). História natural, ecologia e conservação de algumas espécies de plantas e animais da Amazônia (pp. 283-289), Manaus, Edua/Edelbra.

Silva, A.B. \& Marmontel, M. (2009) Ingestão de lixo plástico como provável causa mortis de peixe-boi amazônico (Trichechus inunguis Natterer, 1883), Uakari, 5(1), 105-112.

Snowdon, C.T. (1999) O significado da pesquisa em comportamento animal, Estudos de Psicologia, 4(2), 365-373. 